\title{
Reachable Set Estimation for Discrete-Time Systems with Interval Time-Varying Delays and Bounded Disturbances
}

\author{
Jiemei Zhao ${ }^{1}$ and Yin Sheng ${ }^{2}$ \\ ${ }^{1}$ School of Mathematics and Computer Science, Wuhan Polytechnic University, Wuhan 430023, China \\ ${ }^{2}$ School of Automation, Huazhong University of Science and Technology, Wuhan 430074, China \\ Correspondence should be addressed to Jiemei Zhao; jiemeizhao@163.com
}

Received 1 October 2016; Accepted 23 October 2016

Academic Editor: Xian Zhang

Copyright (c) $2016 \mathrm{~J}$. Zhao and Y. Sheng. This is an open access article distributed under the Creative Commons Attribution License, which permits unrestricted use, distribution, and reproduction in any medium, provided the original work is properly cited.

\begin{abstract}
The reachable set estimation problem for discrete-time systems with delay-range-dependent and bounded disturbances is investigated. A triple-summation term, the upper bound, and the lower bound of time-varying delay are introduced into the Lyapunov function. In this case, an improved delay-range-dependent criterion is established for the addressed problem by constructing the appropriate Lyapunov functional, which guarantees that the reachable set of discrete-time systems with timevarying delay and bounded peak inputs is contained in the ellipsoid. It is worth mentioning that the initial value of the system does not need to be zero. Then, the reachable set estimation problem for time-delay systems with polytopic uncertainties is investigated. The effectiveness and the reduced conservatism of the derived results are demonstrated by an illustrative example.
\end{abstract}

\section{Introduction}

The problem of reachable set estimation has been an important research area in control theory and has extensive applications in many areas, such as safety inspection of system [1], peak-to-peak gain minimization [2], control systems with actuator saturation [3, 4], parameter estimation [5], and other areas. Because time delays cannot be avoided in practical control systems and they cause undesirable dynamic behaviors such as oscillation and instability [6-10], in this context, it is natural to ask what about the reachable set of systems with time delays.

The reachable set estimation problem for time-delay systems has received considerable attention in recent years, such as linear systems with state delays [11-19], linear systems in the presence of both discrete and distributed delays [20,21], and time-varying delay singular systems [22]. However, the considered systems in literatures [11-22] are all continuous. Discrete-time time-delay systems are an important class of dynamic systems because most control engineering application systems are digital implementation. Hence, control design for discrete-time model directly is more convenient. To the best of our knowledge, few efforts have been taken to the reachable set estimation problem of discrete-time systems. Very recently, the paper [23] addresses the problem of reachable set bounding for linear discrete-time systems that are subject to state delay and bounded disturbances. A new idea of minimizing the projection distances of the ellipsoids on each axis was proposed. The reachable set estimation problem for discrete-time polytopic systems with bounded disturbances and multiple constant delays has been studied in [24]. It provides a new method to investigate the problem of reachable set estimation. However, in $[23,24]$, some useful terms were ignored in the Lyapunov function and the derivation process. The ignorance terms may lead to considerable conservativeness. In addition, the literatures above [11-24] all suppose that the initial value of the system is zero. This condition brings some constraints in the process of estimating the bound of reachable set. Therefore, the reachable set estimation problem for discrete-time timevarying delays systems without restrictions on initial value still remains open, which motivates the present study.

In this paper, we aim to study the reachable set bounding for discrete-time linear systems with interval time-varying delays and bounded disturbances. The main contributions 
of this paper lie in three aspects. Firstly, a new delay-rangedependent analysis result is established for discrete-time time-delay systems by retaining some useful terms and the triple-summation term in the difference of the Lyapunov function. The relationship among the time-varying delay, its upper bound, and lower bound is considered. Secondly, the initial value of the system does not need to be zero. Finally, the reachable set estimation problem for polytopic time-varying systems is investigated. A numerical example is given to illustrate the effectiveness of the obtained results.

\section{System Description and Preliminaries}

Consider the following discrete-time singular systems with interval time-varying delay and disturbances:

$$
\begin{aligned}
x(k+1) & =A x(k)+A_{d} x(k-d(k))+B \omega(k), \\
x(k) & =\phi(k), \quad k \in\left[-d_{2}, 0\right],
\end{aligned}
$$

where $x(k)$ is the state vector and $\phi(k)$ is the initial condition; $d(k)$ is a time-varying delay satisfying $0 \leq d_{1} \leq d(k) \leq$ $d_{2}$, where $d_{1}$ and $d_{2}$ are prescribed nonnegative integers representing the lower and upper bounds of the time delay, respectively. $A, A_{d}$, and $B$ are known real constant matrices of appropriate dimensions; $\omega(k)$ is the disturbance which satisfies

$$
\omega^{T}(k) \omega(k) \leq \bar{\omega}^{2},
$$

where $\bar{\omega}$ is a real constant.

A reachable set for system (1) subject to bounded disturbance (2) is defined as

$$
\begin{aligned}
\mathscr{R}_{x} & :=\left\{x(k) \in \mathbf{R}^{n} \mid x(k), \omega(k) \text { satisfy (1) and }(2), k\right. \\
& \geq 0\} .
\end{aligned}
$$

For a matrix $P>0$, we define an ellipsoid $\varepsilon(P, 1)$ bounding the reachable set (3) as follows:

$$
\varepsilon(P, 1):=\left\{x \in \mathbf{R}^{n} \mid x^{T} P x \leq 1\right\} .
$$

Before moving on, we give some definitions and lemmas which will be used in the proof of the main results.

Lemma 1. Let $V(x(k))$ be a positive-definite function: $V\left(x\left(k_{0}\right)\right) \leq \beta \bar{\omega}^{2} /(1-\alpha), k_{0}>0, \alpha \in(0,1)$, and $\beta>0$. If

$$
\Delta V(k)+(1-\alpha) V(k)-\beta \omega^{T}(k) \omega(k) \leq 0,
$$

then $V(k) \leq \beta \bar{\omega}^{2} /(1-\alpha), \forall k \geq k_{0}$.

Proof. By (2) and (5), we have

$$
\Delta V(k)+(1-\alpha) V(k) \leq \beta \bar{\omega}^{2} ;
$$

that is

$$
\Delta\left(V(k)-\frac{\beta \bar{\omega}^{2}}{1-\alpha}\right) \leq-(1-\alpha)\left(V(k)-\frac{\beta \bar{\omega}^{2}}{1-\alpha}\right)
$$

Let $U(k)=V(k)-\beta \bar{\omega}^{2} /(1-\alpha)$. Then, (7) is equivalent to $\Delta U(k) \leq-(1-\alpha) U(k)$. Furthermore,

$$
U(k) \leq \alpha U(k-1) \leq \cdots \leq \alpha^{k-k_{0}} U\left(k_{0}\right) .
$$

Since $\alpha \in(0,1), U\left(k_{0}\right) \leq 0$, and $U(k)=V(k)-\beta \bar{\omega}^{2} /(1-\alpha)$, $V(k) \leq \beta \bar{\omega}^{2} /(1-\alpha), k \geq k_{0}$.

In order to use Lemma 1 conveniently, Lemma 1 can be rewritten as the following form by $\beta=(1-\alpha) / \bar{\omega}^{2}$.

Lemma 2. Let $V(x(k))$ be a positive-definite function: $V\left(x\left(k_{0}\right)\right) \leq 1, k_{0}>0$. If there exists a scalar $\alpha \in(0,1)$ such that

$$
V(k+1)-\alpha V(k)-\frac{1-\alpha}{\bar{\omega}^{2}} \omega^{T}(k) \omega(k) \leq 0,
$$

then $V(k) \leq 1, \forall k \geq k_{0}$.

Remark 3. The reachable set estimation problem is investigated in [11-23] under the condition that the initial values of the system states are zero. However, the condition is removed in Lemma 1. The reachable sets defined in (3) of system (1) can be bounded if $V\left(x\left(k_{0}\right)\right) \leq \beta \bar{\omega}^{2} /(1-\alpha), k_{0}>0$. Let $\beta=(1-\alpha) / \bar{\omega}^{2}$. Lemmas 1 and 2 reduce to (Lemma 2, [23]) and (Lemma 2, [24]), respectively. Therefore, Lemmas 1 and 2 provide more general results for the problem of reachable set estimation.

Lemma 4 (see [24]). Give a positive integer $h \in \mathbf{Z}_{+}$, a scalar $\alpha \in(0,1)$, a vector function $v(k), k \in \mathbf{Z}$, and a matrix $Z>0$. Then, the following inequalities hold:

$$
\begin{aligned}
& \text { (i) }-\sum_{j=h}^{-1} \alpha^{-j} v^{T}(k+j) Z v(k+j) \leq-\widehat{\alpha}\left[\sum_{j=h}^{-1} v^{T}(k+\right. \\
& j)]^{T} Z\left[\sum_{j=h}^{-1} v^{T}(k+j)\right] \text {, where } \widehat{\alpha}=\alpha^{h}(1-\alpha) /\left(1-\alpha^{h}\right) . \\
& \text { (ii) }-\sum_{l=-d}^{-1} \sum_{j=h}^{-1} \alpha^{-j} v^{T}(k+j) Z v(k+j) \leq \\
& -\widetilde{\alpha}\left[\sum_{l=-d}^{-1} \sum_{j=h}^{-1} v^{T}(k+j)\right]^{T} Z\left[\sum_{l=-d}^{-1} \sum_{j=h}^{-1} v^{T}(k+j)\right], \\
& \text { where } \widetilde{\alpha}=\alpha^{d}(1-\alpha)^{2} /\left(1-(d+1) \alpha^{d}+d \alpha^{d+1}\right) .
\end{aligned}
$$

The aim in this paper is to find the intersection of ellipsoids $\varepsilon(P, 1)$ to bound the reachable set defined as (3). Throughout in this paper, $\alpha \in(0,1), d_{12}=d_{2}-d_{1}, \widehat{\alpha}_{1}=\alpha^{d_{1}}(1-\alpha) /\left(1-\alpha^{d_{1}}\right)$, $\widehat{\alpha}_{2}=\alpha^{d_{12}}(1-\alpha) /\left(1-\alpha^{d_{12}}\right), \widetilde{\alpha}_{1}=\alpha^{d_{1}}(1-\alpha)^{2} /\left(1-\left(d_{1}+1\right) \alpha^{d_{1}}+\right.$ $\left.d_{1} \alpha^{d_{1}+1}\right)$, and $\widetilde{\alpha}_{2}=\alpha^{d_{12}}(1-\alpha)^{2} /\left(1-\left(d_{12}+1\right) \alpha^{d_{12}}+d_{12} \alpha^{d_{12}+1}\right)$. Then, the main results are given.

\section{Reachable Set Estimation for Nominal Systems}

Theorem 5. Consider system (1) with the input satisfying (2). If there exist matrices $P>0, Q_{1}>0, Q_{2}>0, Q_{3}>0, Z_{1}>$ $0, Z_{2}>0, R_{1}$, and $R_{2}$ and a scalar $\alpha \in(0,1)$ such that the following LMI holds,

$$
\Psi=\left[\begin{array}{ccc}
\Phi_{11} & \Phi_{12} P & \Phi_{13} W \\
\star & -P & 0 \\
\star & \star & -W
\end{array}\right]<0,
$$




$$
\begin{aligned}
& \Phi_{11}=\left[\begin{array}{ccccccc}
\Delta_{11} & 0 & \widehat{\alpha}_{1} Z_{1} & 0 & \tilde{\alpha}_{1} d_{1} R_{1} & \widetilde{\alpha}_{2} d_{12} R_{2} & 0 \\
\star & \alpha^{d_{2}} Q_{3} & 0 & 0 & 0 & 0 & 0 \\
\star & \star & \Delta_{33} & \widehat{\alpha}_{2} Z_{2} & 0 & 0 & 0 \\
\star & \star & \star & -\alpha^{d_{2}} Q_{2}-\widehat{\alpha}_{2} Z_{2} & 0 & 0 & 0 \\
\star & \star & \star & \star & -\widetilde{\alpha}_{1} R_{1} & 0 & 0 \\
\star & \star & \star & \star & \star & -\widetilde{\alpha}_{2} R_{2} & 0 \\
\star & \star & \star & \star & \star & \star & -\frac{1-\alpha}{\bar{\omega}^{2}} I
\end{array}\right], \\
& \Delta_{11}=-\alpha P+Q_{1}+Q_{2}+Q_{3}-\widehat{\alpha}_{1} Z_{1}-d_{1}^{2} \widetilde{\alpha}_{1} R_{1}-d_{12}^{2} \widetilde{\alpha}_{2} R_{2}, \\
& \Delta_{33}=-\alpha^{d_{1}} Q_{1}-\widehat{\alpha}_{1} Z_{1}-\widehat{\alpha}_{2} Z_{2} \text {, } \\
& \Phi_{12}=\left[\begin{array}{lllllll}
A & A_{d} & 0 & 0 & 0 & 0 & B
\end{array}\right]^{T} \text {, } \\
& \Phi_{13}=\left[\begin{array}{lllllll}
A-I & A_{d} & 0 & 0 & 0 & 0 & B
\end{array}\right]^{T} \text {, } \\
& W=d_{1} Z_{1}+d_{12} Z_{2}+\frac{d_{1}\left(1+d_{1}\right)}{2} R_{1}+\frac{d_{12}\left(d_{1}+d_{2}+1\right)}{2} R_{2} \text {, }
\end{aligned}
$$

then the reachable set of system (1) is contained in the ellipsoid $\varepsilon(P, 1)$.

Proof. Define $\eta(k)=x(k+1)-x(k)$. Then, we construct the following Lyapunov functional:

$$
\begin{aligned}
V(k)= & x^{T}(k) P x(k)+\sum_{s=k-d_{1}}^{k-1} \alpha^{k-s-1} x^{T}(s) Q_{1} x(s) \\
& +\sum_{s=k-d_{2}}^{k-1} \alpha^{k-s-1} x^{T}(s) Q_{2} x(s) \\
& +\sum_{s=k-d(k)}^{k-1} \alpha^{k-s-1} x^{T}(s) Q_{3} x(s) \\
& +\sum_{j=-d_{1}}^{-1} \sum_{s=k+j}^{k-1} \alpha^{k-s-1} \eta^{T}(s) Z_{1} \eta(s)+d_{12} \\
& \cdot \sum_{j=-d_{2}} \sum_{s=k+j}^{k-1} \alpha^{k-s-1} \eta^{T}(s) Z_{2} \eta(s) \\
& +\sum_{l=-d_{1}}^{-1} \sum_{j=l}^{-1} \sum_{s=k+j}^{k-1} \alpha^{k-s-1} \eta^{T}(s) R_{1} \eta(s)+d_{12} \\
& \cdot \sum_{l=-d_{1}-1} \sum_{j=l}^{-1} \sum_{s=k+j}^{k-1} \alpha^{k-s-1} \eta^{T}(s) R_{2} \eta(s)
\end{aligned}
$$

In the following, we will prove that $V(x(k)) \leq 1$ under the condition in (10).
It is not difficult to obtain that

$$
\begin{aligned}
J= & V(k+1)-\alpha V(k)-\frac{1-\alpha}{\bar{\omega}^{2}} \omega^{T}(k) \omega(k) \\
= & x^{T}(k+1) P x(k+1)-\alpha x^{T}(k) P x(k) \\
& -\frac{1-\alpha}{\bar{\omega}^{2}} \omega^{T}(k) \omega(k)+\sum_{s=k+1-d_{1}}^{k} \alpha^{k-s} x^{T}(s) Q_{1} x(s) \\
& -\sum_{s=k-d_{1}}^{k-1} \alpha^{k-s} x^{T}(s) Q_{1} x(s) \\
& +\sum_{s=k+1-d_{2}}^{k} \alpha^{k-s} x^{T}(s) Q_{2} x(s) \\
& -\sum_{s=k-d_{2}}^{k-1} \alpha^{k-s} x^{T}(s) Q_{2} x(s) \\
& +\sum_{s=k-d(k)}^{k} \alpha^{k-s} x^{T}(s) Q_{3} x(s) \\
& \alpha^{k-s} x^{T}(s) Q_{3} x(s) \\
& -k(k)
\end{aligned}
$$$$
+\sum_{j=-d_{1}}^{-1} \sum_{s=k+1+j}^{k} \alpha^{k-s} \eta^{T}(s) Z_{1} \eta(s)
$$ 


$$
\begin{aligned}
& -\sum_{j=-d_{1}}^{-1} \sum_{s=k+j}^{k-1} \alpha^{k-s} \eta^{T}(s) Z_{1} \eta(s) \\
& +\sum_{j=-d_{2}}^{-d_{1}-1} \sum_{s=k+1+j}^{k} \alpha^{k-s} \eta^{T}(s) Z_{2} \eta(s) \\
& -\sum_{j=-d_{2}}^{-d_{1}-1} \sum_{s=k+j}^{k-1} \alpha^{k-s} \eta^{T}(s) Z_{2} \eta(s) \\
& +\sum_{l=-d_{1}}^{-1} \sum_{j=l}^{-1} \sum_{s=k+1+j}^{k} \alpha^{k-s} \eta^{T}(s) R_{1} \eta(s) \\
& -\sum_{l=-d_{1}}^{-1} \sum_{j=l}^{-1} \sum_{s=k+j}^{k-1} \alpha^{k-s} \eta^{T}(s) R_{1} \eta(s) \\
& +\sum_{j=-d_{2}}^{-d_{1}-1} \sum_{j=l}^{-1} \sum_{s=k+1+j}^{k} \alpha^{k-s} \eta^{T}(s) R_{2} \eta(s) \\
& -\sum_{j=-d_{2}}^{-d_{1}-1} \sum_{j=l}^{-1} \sum_{s=k+j}^{k-1} \alpha^{k-s} \eta^{T}(s) R_{2} \eta(s) \\
& =x^{T}(k+1) E^{T} P E x(k+1)+\eta^{T}(k) W \eta(k) \\
& -\frac{1-\alpha}{\bar{\omega}^{2}} \omega^{T}(k) \omega(k) \\
& +x^{T}(k)\left(-\alpha P+Q_{1}+Q_{2}+Q_{3}\right) x(k) \\
& -\alpha^{d_{1}} x^{T}\left(k-d_{1}\right) Q_{1} x\left(k-d_{1}\right) \\
& -\alpha^{d_{2}} x^{T}\left(k-d_{2}\right) Q_{2} x\left(k-d_{2}\right) \\
& -\alpha^{d(k)} x^{T}(k-d(k)) Q_{3} x(k-d(k)) \\
& -\sum_{j=-d_{1}}^{-1} \alpha^{-j} \eta^{T}(k+j) Z_{1} \eta(k+j) \\
& -\sum_{j=-d_{2}}^{-d_{1}-1} \alpha^{-j} \eta^{T}(k+j) Z_{2} \eta(k+j) \\
& -\sum_{l=-d_{1}}^{-1} \sum_{j=l}^{-1} \alpha^{-j} \eta^{T}(k+j) R_{1} \eta(k+j) \\
& -\sum_{l=-d_{2}}^{-d_{1}-1} \sum_{j=l}^{-1} \alpha^{-j} \eta^{T}(k+j) R_{2} \eta(k+j) .
\end{aligned}
$$

Applying Lemma 4, then

$$
\begin{aligned}
J \leq & x^{T}(k+1) P x(k+1)+\eta^{T}(k) W \eta(k)-\frac{1-\alpha}{\bar{\omega}^{2}} \\
& \cdot \omega^{T}(k) \omega(k)+x^{T}(k)\left(-\alpha P+Q_{1}+Q_{2}+Q_{3}\right) x(k)
\end{aligned}
$$

$$
\begin{aligned}
& -\alpha^{d_{1}} x^{T}\left(k-d_{1}\right) Q_{1} x\left(k-d_{1}\right)-\alpha^{d_{2}} x^{T}\left(k-d_{2}\right) \\
& \text { - } Q_{2} x\left(k-d_{2}\right)-\alpha^{d_{2}} x^{T}(k-d(k)) Q_{3} x(k-d(k)) \\
& -\widehat{\alpha}_{1}\left[x(k)-x\left(k-d_{1}\right)\right]^{T} Z_{1}\left[x(k)-x\left(k-d_{1}\right)\right] \\
& -\widehat{\alpha}_{2}\left[x\left(k-d_{1}\right)-x\left(k-d_{2}\right)\right]^{T} \\
& \cdot Z_{2}\left[x\left(k-d_{1}\right)-x\left(k-d_{2}\right)\right] \\
& -\widetilde{\alpha}_{1}\left[d_{1} x(k)-\sum_{l=-d_{1}}^{-1} x(k+l)\right]^{T} \\
& \cdot R_{1}\left[d_{1} x(k)-\sum_{l=-d_{1}}^{-1} x(k+l)\right] \\
& -\widetilde{\alpha}_{2}\left[d_{12} x(k)-\sum_{l=-d_{2}}^{-d_{1}-1} x(k+l)\right]^{T} \\
& \cdot R_{2}\left[d_{12} x(k)-\sum_{l=-d_{2}}^{-d_{1}-1} x(k+l)\right]=\xi^{T}(k) \\
& \cdot\left(\Phi_{11}+\Phi_{12} P \Phi_{12}^{T}+\Phi_{13} W \Phi_{13}^{T}\right) \xi(k)
\end{aligned}
$$

where $\xi(k)=\left[x^{T}(k) x^{T}(k-d(k)) x^{T}\left(k-d_{1}\right) x^{T}(k-\right.$ $\left.\left.d_{2}\right) \quad \sum_{s=1}^{d_{1}} x^{T}(k-s) \quad \sum_{s=d_{1}+1}^{d_{2}} x^{T}(k-s) \quad \omega^{T}(k)\right]^{T}$.

Applying the Schur complement, (10) is equivalent to $\Phi_{11}+\Phi_{12} P \Phi_{12}^{T}+\Phi_{13} W \Phi_{13}^{T}<0$. This, together with (14), ensures that $J \leq \xi^{T}(k) \Psi \xi(k)<0$. Then, by using Lemma 2, we have that $V(x(t)) \leq 1$. Based on (19), we obtain $x^{T}(k) P x(k) \leq$ 1 . This means that the state trajectories of system (1) starting from the origin are bounded within the ellipsoid $\varepsilon(P, 1)$.

Remark 6. Under the assumptions that the initial conditions of the systems are zero and state trajectories start from the origin, the reachable set bounding problems are investigated in $[23,24]$. The assumptions are removed in Theorem 5. In this case, the methods in $[23,24]$ are invalid when the initial conditions $\phi(k) \neq 0$. Therefore, our results are more general.

By using Theorem 5, a corollary can be obtained directly.

Corollary 7. Consider the system in (1) with $d(k)=d$. If there exist matrices $P>0, Q_{1}>0, Q_{2}>0, Q_{3}>0, Z>0$, and $R$ and a scalar $\alpha \in(0,1)$ such that the following LMI holds,

$$
\Psi=\left[\begin{array}{ccc}
\Phi_{11} & \Phi_{12} P & \Phi_{13} W \\
\star & -P & 0 \\
\star & \star & -W
\end{array}\right]<0,
$$


where

$$
\begin{aligned}
& \Phi_{11}=\left[\begin{array}{ccccc}
\Delta_{11} & 0 & -\widehat{\alpha}_{1} Z & \widetilde{\alpha}_{1} d R & 0 \\
\star & \alpha^{d_{2}} Q_{3} & 0 & 0 & 0 \\
\star & \star & \Delta_{33} & 0 & 0 \\
\star & \star & \star & -\widetilde{\alpha}_{1} R & 0 \\
\star & \star & \star & \star & -\frac{1-\alpha}{\bar{\omega}^{2}} I
\end{array}\right], \\
& \Delta_{11}=-\alpha P+Q_{3}-\widehat{\alpha}_{1} Z-d_{1}^{2} \widetilde{\alpha}_{1} R, \\
& \Delta_{33}=-\alpha^{d} Q_{1}-\widehat{\alpha}_{1} Z, \\
& \Phi_{12}=\left[\begin{array}{lllll}
A & A_{d} & 0 & 0 & B
\end{array}\right]^{T}, \\
& \Phi_{13}=\left[\begin{array}{lllll}
A-I & A_{d} & 0 & 0 & B
\end{array}\right]^{T}, \\
& W=d Z+\frac{d(1+d)}{2} R,
\end{aligned}
$$

then the reachable set of system (1) with $d(k)=d$ is contained in the ellipsoid $\varepsilon(P, 1)$.

\section{Reachable Set Estimation for Uncertain Systems}

If there exist polytopic uncertainties in system matrices $A$, $A_{d}$, and $B$, that is,

$$
\begin{aligned}
\Lambda & =\left[\begin{array}{lll}
A & A_{d} & B
\end{array}\right], \\
\Lambda & =\sum_{i=1}^{N} \lambda_{i} \Lambda_{i}, \quad \lambda_{i}>0, \\
\sum_{i=1}^{N} \lambda_{i} & =1,
\end{aligned}
$$

where $N$ is the number of polytope vertices and the $N$ vertices of the polytopic are described by $\Lambda_{i}=\left[\begin{array}{lll}A_{i} & A_{d i} & B_{i}\end{array}\right](i=$ $1,2, \ldots, N)$, it is easy to extend Theorem 5 in such a case.

Theorem 8. Consider the system in (1) with polytopic uncertainties (17) and (18). If there exist matrices $P>0, Q_{1}>$ $0, Q_{2}>0, Q_{3}>0, Z_{1}>0, Z_{2}>0, R_{1}$, and $R_{2}$ and a scalar $\alpha \in(0,1)$ such that the following LMI holds,

$$
\Psi=\left[\begin{array}{ccc}
\Phi_{11} & \Phi_{12} P & \Phi_{13} W \\
\star & -P & 0 \\
\star & \star & -W
\end{array}\right]<0,
$$

where

$$
\begin{aligned}
& \Phi_{11}=\left[\begin{array}{ccccccc}
\Delta_{11} & 0 & \widehat{\alpha}_{1} Z_{1} & 0 & \widetilde{\alpha}_{1} d_{1} R_{1} & \widetilde{\alpha}_{2} d_{12} R_{2} & 0 \\
\star & \alpha^{d_{2}} Q_{3} & 0 & 0 & 0 & 0 & 0 \\
\star & \star & \Delta_{33} & \widehat{\alpha}_{2} Z_{2} & 0 & 0 & 0 \\
\star & \star & \star & -\alpha^{d_{2}} Q_{2}-\widehat{\alpha}_{2} Z_{2} & 0 & 0 & 0 \\
\star & \star & \star & \star & -\widetilde{\alpha}_{1} R_{1} & 0 & 0 \\
\star & \star & \star & \star & \star & -\widetilde{\alpha}_{2} R_{2} & 0 \\
\star & \star & \star & \star & \star & \star & -\frac{1-\alpha}{\bar{\omega}^{2}} I
\end{array}\right], \\
& \Delta_{11}=-\alpha P+Q_{1}+Q_{2}+Q_{3}-\widehat{\alpha}_{1} Z_{1}-d_{1}^{2} \widetilde{\alpha}_{1} R_{1}-d_{12}^{2} \widetilde{\alpha}_{2} R_{2}, \\
& \Delta_{33}=-\alpha^{d_{1}} Q_{1}-\widehat{\alpha}_{1} Z_{1}-\widehat{\alpha}_{2} Z_{2}, \\
& \Phi_{12}=\left[\begin{array}{lllllll}
A_{i} & A_{d i} & 0 & 0 & 0 & 0 & B_{i}
\end{array}\right]^{T}, \\
& \Phi_{13}=\left[\begin{array}{lllllll}
A_{i}-I & A_{d i} & 0 & 0 & 0 & 0 & B_{i}
\end{array}\right]^{T}, \\
& W=d_{1} Z_{1}+d_{12} Z_{2}+\frac{d_{1}\left(1+d_{1}\right)}{2} R_{1}+\frac{d_{12}\left(d_{1}+d_{2}+1\right)}{2} R_{2},
\end{aligned}
$$


then, for any time-varying delay $d(k)$ satisfying (2), the reachable sets of system (1) with polytopic uncertainties (17) can be bounded by the ellipsoid $\varepsilon(P, 1)$.

Corollary 9. Consider the system in (1) with polytopic uncertainties (17) and $d(k)=d$. If there exist matrices $P>0, Q_{1}>$ $0, Q_{2}>0, Q_{3}>0, Z>0, R$ and a scalar $\alpha \in(0,1)$ such that the following LMI holds,

$\Psi$

$$
\begin{aligned}
& {\left[\begin{array}{ccccccc}
\Delta_{11} & 0 & -\widehat{\alpha}_{1} Z & \widetilde{\alpha} d_{1} R & 0 & A_{i} P & \left(A_{i}-I\right) W \\
\star & \alpha^{d_{2}} Q_{3} & 0 & 0 & 0 & A_{d i} P & A_{d i} W \\
\star & \star & \Delta_{33} & 0 & 0 & 0 & 0 \\
\star & \star & \star & -\widetilde{\alpha} R & 0 & 0 & 0 \\
\star & \star & \star & \star & -\frac{1-\alpha}{\bar{\omega}^{2}} I & B_{i} P & B_{i} W \\
\star & \star & \star & \star & \star & -P & 0 \\
\star & \star & \star & \star & \star & \star & -W
\end{array}\right]} \\
& <0,
\end{aligned}
$$

where

$$
\begin{aligned}
\Delta_{11} & =-P+Q_{3}-\widehat{\alpha}_{1} Z-d_{1}^{2} \widetilde{\alpha}_{1} R, \\
\Delta_{33} & =-\alpha^{d_{1}} Q_{1}-\widehat{\alpha}_{1} Z, \\
W & =d_{1} Z+\frac{d_{1}\left(1+d_{1}\right)}{2} R,
\end{aligned}
$$

then the reachable sets of system (1) can be bounded by the ellipsoid $\varepsilon(P, 1)$.

Remark 10. To find the "smallest" bound for the reachable set, one may propose a simple optimisation problem. That is, maximise $\delta$ subject to $\delta I \leq \bar{P}$, which can be transformed to the following optimisation problem:

$$
\begin{array}{ll}
\text { minimize } & \widehat{\delta}\left(\widehat{\delta}=\delta^{-1}\right) \\
\text { subject to } & \text { (a) }\left[\begin{array}{cc}
\bar{\delta} I & I \\
I & \widehat{P}
\end{array}\right] \geq 0
\end{array}
$$

(b) Equations: (10) or (19).

\section{Numerical Example}

Example 1. Consider discrete-time time-varying delay system (1) with

$$
\begin{aligned}
A & =\left[\begin{array}{cc}
0.21 & -0.01 \\
-0.9 & 0.1
\end{array}\right], \\
A_{d} & =\left[\begin{array}{cc}
-0.02 & 0.01 \\
-0.2 & -0.01
\end{array}\right], \\
B & =\left[\begin{array}{c}
0.1 \\
0.15
\end{array}\right]
\end{aligned}
$$

TABLE 1: Different values of $\bar{\delta}$ and $P$ by choosing different $\alpha$.

\begin{tabular}{cccc}
\hline$\alpha$ & $\bar{\delta}$ in Theorem 5 & \multicolumn{1}{c}{$P$} & $\bar{\delta}$ in $[23,24]$ \\
\hline 0.4 & 0.4014 & {$\left[\begin{array}{cc}27.5819 & -1.1019 \\
-1.1019 & 1.4983\end{array}\right]$} & - \\
0.6 & 0.1560 & {$\left[\begin{array}{cc}35.1870 & -4.3501 \\
-4.3501 & 4.5431\end{array}\right]$} & - \\
0.8 & 0.2206 & {$\left[\begin{array}{cc}20.3993 & -3.7143 \\
-3.7143 & 3.4333\end{array}\right]$} & -
\end{tabular}

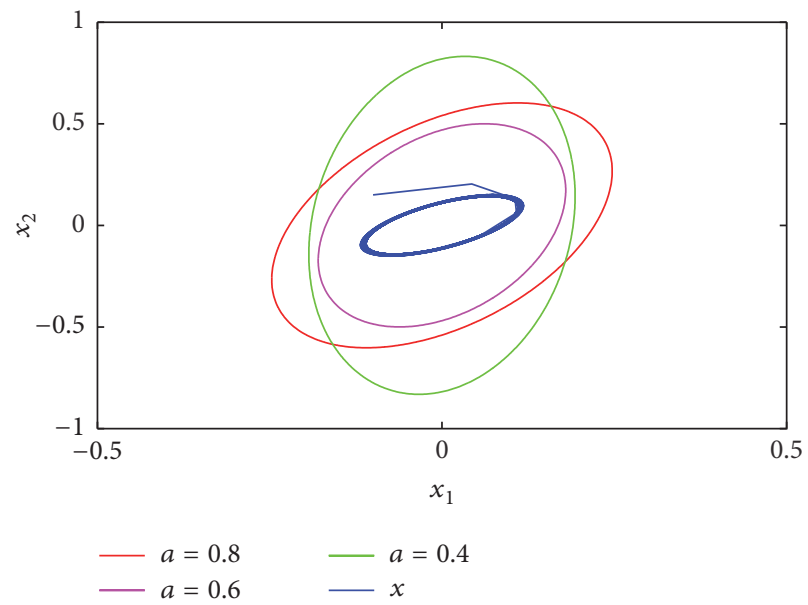

FIGURE 1: The reachable sets and ellipsoidal bounds.

and $\omega(k)=\sin (7 k)$. Let $d_{1}=2$ and $d_{2}=4$. Assume the initial state is $\phi(t)=\left[\begin{array}{ll}-0.1 & 0.15\end{array}\right]^{T}$. Since the initial condition is not zero, the methods in paper $[23,24]$ are invalid. By solving LMI (10) of Theorem 5 , the values $\bar{\delta}$ for different $\alpha$ by LMI toolbox are given in Table 1. Meanwhile, corresponding ellipsoidal bounds of the reachable sets are depicted in Figure 1. It shows that the reachable set of system (1) can be bounded by the obtained ellipsoid $\varepsilon(P, 1)$.

\section{Conclusions}

In this paper, the problem of reachable set estimation for discrete-time systems with interval time-varying delays and bounded disturbances has been investigated. By introducing triple-summation terms, a novel Lyapunov function is constructed. Then, a delay-range-dependent criterion is established and the initial condition of discrete-time timevarying delay system is not required to be zero. Based on this result, the reachable set estimation problem for polytopic time-varying systems is investigated. The effectiveness of the obtained results has been verified through a numerical example. 


\section{Competing Interests}

The authors declare that they have no competing interests.

\section{Acknowledgments}

This work is supported by the Natural Science Foundation of Hubei Province of China (2016CFB273), the Research and Innovation Initiatives of WHPU (2015d9), and the Scientific Research Foundation of Wuhan Polytechnic University Introduction (cultivate) Talents (2014RZ04).

\section{References}

[1] S. P. Boyd, L. El Ghaoui, E. Feron, and V. Balakrishnan, Linear Matrix Inequalities in System and Control Theory, vol. 15 of SIAM Studies in Applied Mathematics, SIAM, Philadelphia, Pa, USA, 1994.

[2] J. Abedor, K. Nagpal, and K. Poolla, "A linear matrix inequality approach to peak-to-peak gain minimization," International Journal of Robust and Nonlinear Control, vol. 6, no. 9-10, pp. 899-927, 1996.

[3] T. Hu and Z. Lin, Control Systems with Actuator Saturation: Analysis and Design, Birkhäuser, Boston, Mass, USA, 2001.

[4] T. Hu and Z. Lin, "Composite quadratic Lyapunov functions for constrained control systems," IEEE Transactions on Automatic Control, vol. 48, no. 3, pp. 440-450, 2003.

[5] C. Durieu, E. Walter, and B. Polyak, "Multi-input multi-output ellipsoidal state bounding," Journal of Optimization Theory and Applications, vol. 111, no. 2, pp. 273-303, 2001.

[6] X. Zhang, L. Wu, and S. Cui, "An improved integral inequality to stability analysis of genetic regulatory networks with interval time-varying delays," IEEE/ACM Transactions on Computational Biology and Bioinformatics, vol. 12, no. 2, pp. 398-409, 2015.

[7] Y. Sheng, Y. Shen, and M. Zhu, "Delay-dependent global exponential stability for delayed recurrent neural networks," IEEE Transactions on Neural Networks and Learning Systems, 2016.

[8] X. Zhang, L. Wu, and J. Zou, "Globally asymptotic stability analysis for genetic regulatory networks with mixed delays: an M-matrix-based approach," IEEE/ACM Transactions on Computational Biology and Bioinformatics, vol. 13, no. 1, pp. 135-147, 2016.

[9] X. Zhang, Y. Han, L. Wu, and J. Zou, "M-matrix-based globally asymptotic stability criteria for genetic regulatory networks with time-varying discrete and unbounded distributed delays," Neurocomputing, vol. 174, pp. 1060-1069, 2016.

[10] J. Zhao, L. Zhang, and X. Qi, "A necessary and sufficient condition for stabilization of switched descriptor time-delay systems under arbitrary switching," Asian Journal of Control, vol. 18, no. 1, pp. 266-272, 2016.

[11] E. Fridman and U. Shaked, "On reachable sets for linear systems with delay and bounded peak inputs," Automatica, vol. 39, no. 11, pp. 2005-2010, 2003.

[12] J.-H. Kim, "Improved ellipsoidal bound of reachable sets for time-delayed linear systems with disturbances," Automatica, vol. 44, no. 11, pp. 2940-2943, 2008.

[13] Z. Zuo, Y. Chen, Y. Wang, D. W. Ho, M. Z. Chen, and H. Li, "A note on reachable set bounding for delayed systems with polytopic uncertainties," Journal of the Franklin Institute, vol. 350, no. 7, pp. 1827-1835, 2013.

[14] O. M. Kwon, S. M. Lee, and J. H. Park, "On the reachable set bounding of uncertain dynamic systems with time-varying delays and disturbances," Information Sciences, vol. 181, no. 17, pp. 3735-3748, 2011.

[15] P. T. Nam and P. N. Pathirana, "Further result on reachable set bounding for linear uncertain polytopic systems with interval time-varying delays," Automatica, vol. 47, no. 8, pp. 1838-1841, 2011.

[16] B. Zhang, J. Lam, and S. Xu, "Reachable set estimation and controller design for distributed delay systems with bounded disturbances," Journal of the Franklin Institute. Engineering and Applied Mathematics, vol. 351, no. 6, pp. 3068-3088, 2014.

[17] B. Zhang, J. Lam, and S. Xu, "Relaxed results on reachable set estimation of time-delay systems with bounded peak inputs," International Journal of Robust and Nonlinear Control, vol. 26, no. 9, pp. 1994-2007, 2016.

[18] L. V. Hien and H. M. Trinh, "A new approach to state bounding for linear time-varying systems with delay and bounded disturbances," Automatica, vol. 50, no. 6, pp. 1735-1738, 2014.

[19] Y. Sheng and Y. Shen, "Improved reachable set bounding for linear time-delay systems with disturbances," Journal of the Franklin Institute, vol. 353, no. 12, pp. 2708-2721, 2016.

[20] Z. Zuo, D. W. C. Ho, and Y. Wang, "Reachable set estimation for linear systems in the presence of both discrete and distributed delays," IET Control Theory and Applications, vol. 5, no. 15, pp. 1808-1812, 2011.

[21] Z. Zuo, Y. Fu, and Y. Wang, "Results on reachable set estimation for linear systems with both discrete and distributed delays," IET Control Theory and Applications, vol. 6, no. 14, pp. 2346-2350, 2012.

[22] Z. Feng and J. Lam, "On reachable set estimation of singular systems," Automatica, vol. 52, pp. 146-153, 2015.

[23] N. D. That, P. T. Nam, and Q. P. Ha, "Reachable set bounding for linear discrete-time systems with delays and bounded disturbances," Journal of Optimization Theory and Applications, vol. 157, no. 1, pp. 96-107, 2013.

[24] J. Lam, B. Y. Zhang, Y. Chen, and S. Y. Xu, "Reachable set estimation for discrete-time linear systems with time delays," International Journal of Robust and Nonlinear Control, vol. 25, no. 2, pp. 269-281, 2015. 


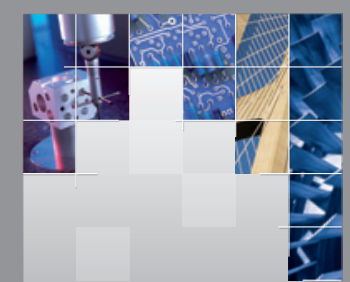

\section{Enfincering}
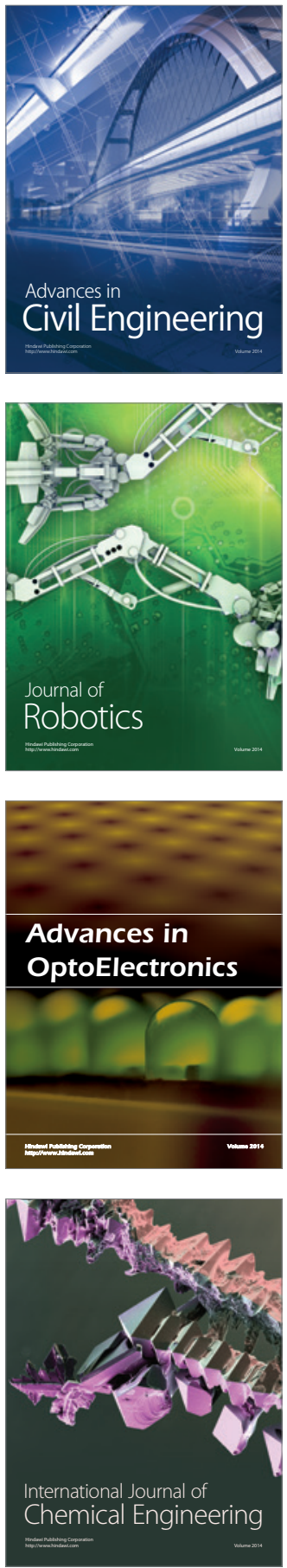

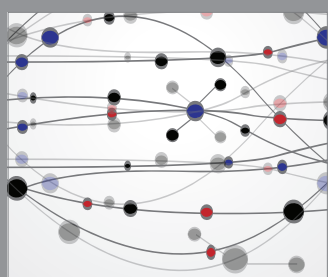

The Scientific World Journal

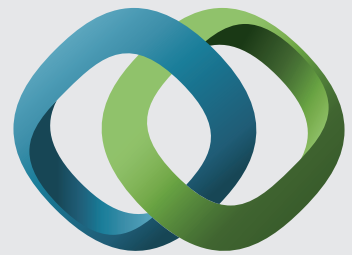

\section{Hindawi}

Submit your manuscripts at

http://www.hindawi.com
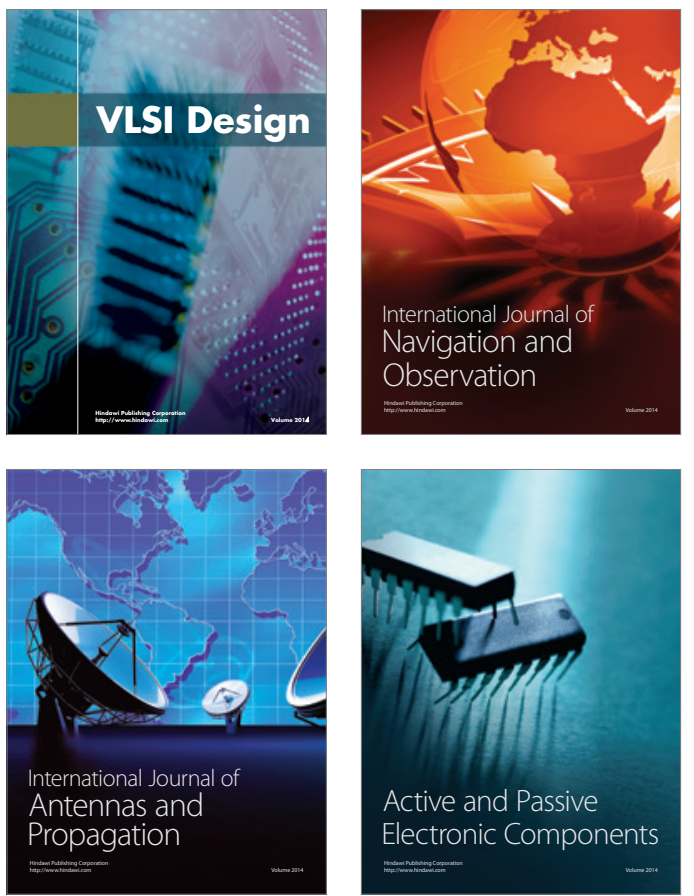
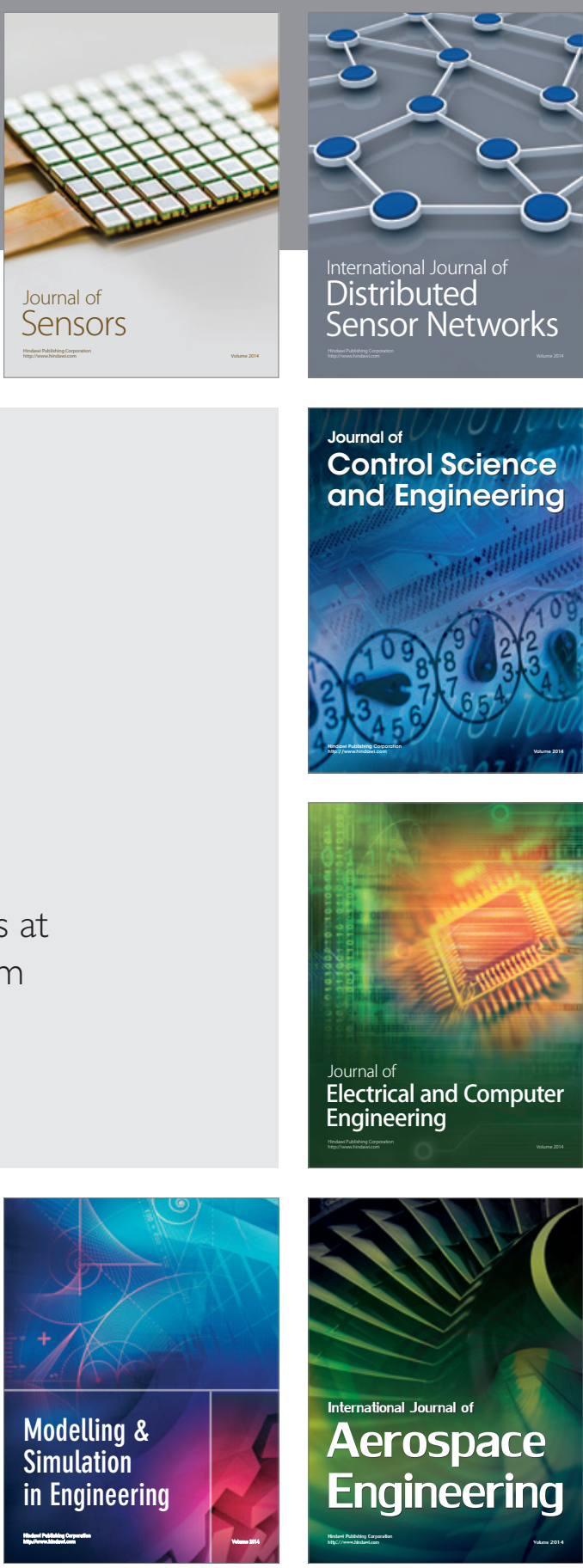

International Journal of

Distributed

Sensor Networks

Journal of

Control Science

and Engineering
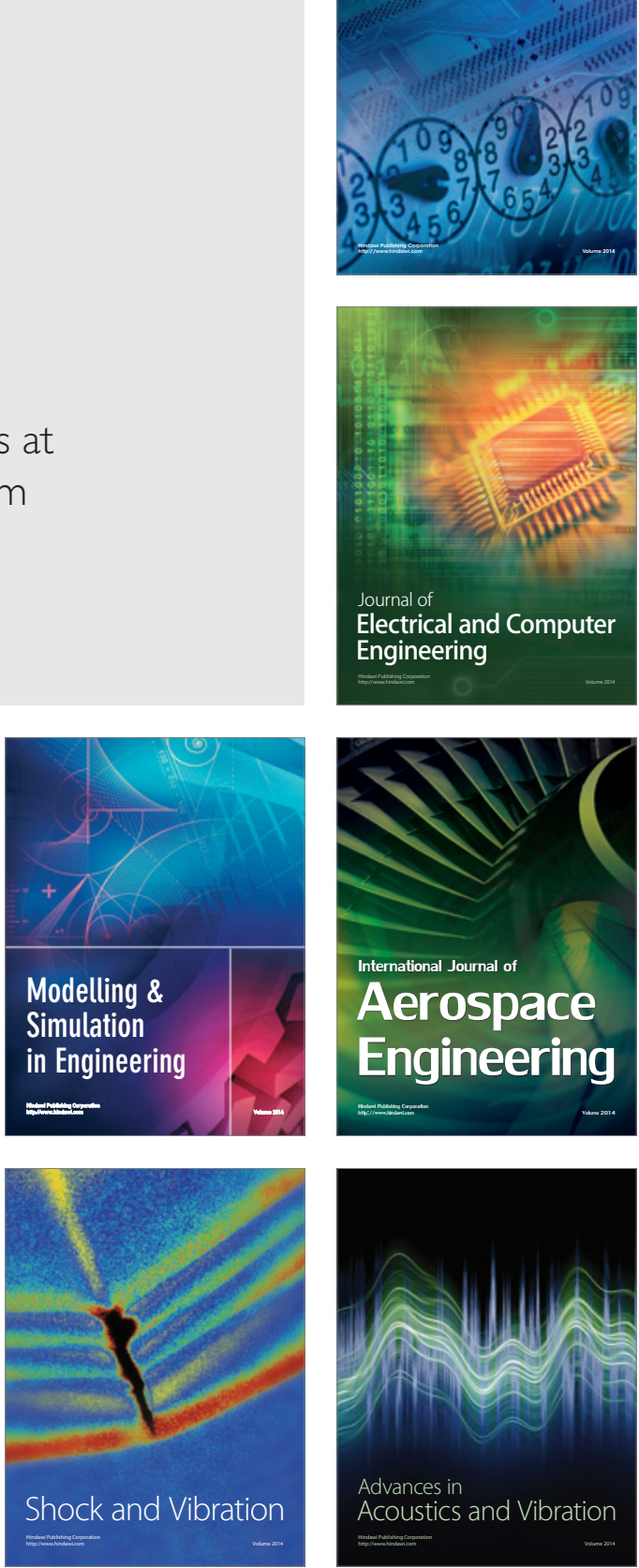